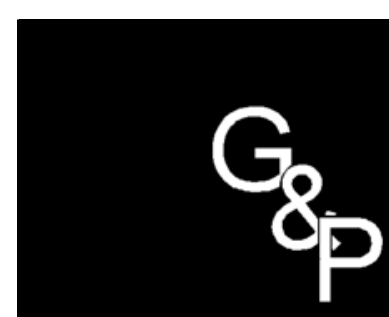

\section{GESTÃO}

$\&$

\section{PRODUÇÃO}

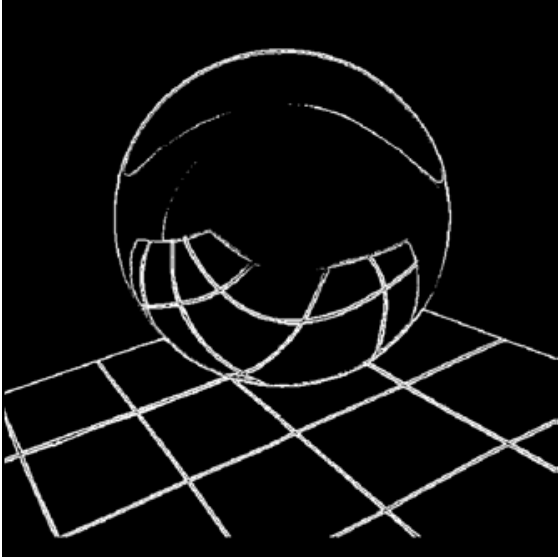

v.7, n.3, p.269-282, dez. 2000

\title{
UM MODELO DE REFERÊNCIA PARA GESTÃO DA PRODUÇÃO EM SISTEMAS DE PRODUÇÃO ASSEMBLY TO ORDER - ATO E SUAS MÚLTIPLAS APLICAÇÕES
}

\author{
Carlos Frederico Bremer \\ Rogério de Paula Lenza \\ Escola de Engenharia de São Carlos - USP \\ Engenharia de Produção Mecânica \\ Av. Dr. Carlos Botelho, 1465 - São Carlos - SP \\ Tel.: (16) 273-9425 \\ E-mails: cfbremer@prod.eesc.sc.usp.br \\ rlenza@prod.eesc.sc.usp.br
}

\section{Resumo}

A busca por competitividade nas empresas direciona cada vez mais para uma estrutura orientada a processos de negócios. Diante disso, os modelos de referência tornam-se relevantes, pois é a partir deles que são mapeados e documentados os processos de negócios. Este artigo apresenta um modelo de referência para o processo de negócio de Gestão da Produção, para empresas que montam seus produtos finais sob encomenda. Esse modelo de referência foi desenvolvido pelo Grupo de Logística Integrada do Núcleo de Manufatura Avançada da USP São Carlos. Primeiramente é apresentado o processo de seleção do método de modelagem, diante dos principais métodos existentes. A seguir é apresentado o processo de desenvolvimento do modelo de referência, bem como as principais atividades e informações que o compõem e, finalmente, é feita uma discussão sobre as possibilidades de uso do modelo apresentando e mostrando uma aplicação para ensino.

Palavras-chave: gestão da produção, modelos de referência, processo de negócio.

\section{Introdução}

s mudanças que vêm ocorrendo no mercado exigem que as empresas adotem uma nova postura de atuação frente aos seus clientes, competidores, fornecedores e em relação à sua administração interna. Diante desse contexto, as empresas investem cada vez mais em ações de melhorias, tais como: processos de reengenharia, adoção de um sistema de gestão empresarial 
integrado, certificações ISO (International Standart Organization), produção enxuta, custeio por atividades, entre outras, cujo objetivo principal é capacitar a empresas para atuar de forma competitiva no mercado.

Porém, cada vez mais tem se discutido o resultado obtido por essas ações. A grande maioria das ações demanda um alto investimento, provoca mudanças no comportamento da empresa, tanto internamente quanto externamente, e por último algumas delas possuem um longo período de implantação, como por exemplo a implantação de sistema de gestão empresarial integrado.

Um aspecto comum quando se pretende adotar uma ação é que a maioria delas exige que sejam levantadas as atividades, funções, informações e recursos que são utilizados pela empresa, ou seja, que sejam levantados os processos de negócios que existem na empresa. Processo de negócio pode ser definido como uma série contínua de atividades realizadas pela empresa, com a finalidade de gerar um produto ou informação (SCHEER, 1998). O ponto de partida e o produto final de um processo de negócio são direcionados a um cliente externo ou interno (SCHEER, 1998). ROZENFELD (1996) define processo de negócio como sendo um fenômeno que ocorre dentro das empresas. Compreende um conjunto de atividades realizadas na empresa, associadas às informações que manipula, utilizando os recursos e a organização da empresa. Forma uma unidade coesa e deve ser focalizado em um tipo de negócio, que normalmente está direcionado a um determinado mercado/cliente, com fornecedores bem definidos.

Porém a atividade de modelagem dos processos de negócio ainda não é uma prática comum entre as empresas, o que acaba contribuindo para o aumento de custo e de tempo de implantação de uma ação ou projeto de melhoria. Isso porque as empresas têm de investir tempo e recursos no desenvolvimento do modelo de seus processos de negócios, uma vez que, se as empresas já possuíssem o modelo, essa atividade não seria necessária.
No sentido de minimizar a falta de um modelo dos processos de negócio nas empresas, algumas organizações de pesquisa, empresas de consultoria e empresas de desenvolvimento de sistemas, desenvolveram, a partir de pesquisas e de suas experiências no mercado, modelos que podem ser utilizados como referências para que as empresas, a partir desses modelos de referência, construam seus próprios modelos. Estudos de casos recentes, mostraram que o uso de modelos de referência em projetos organizacionais podem reduzir o custo e o tempo de implantação em até 30\% (SCHEER, 1998).

O objetivo deste artigo é apresentar um modelo de referência para o processo de negócio de Gestão da Produção, para empresas que montam seus produtos finais sob encomenda. Esse modelo de referência foi desenvolvido pelo grupo de Logística Integrada do Núcleo de Manufatura Avançada da USP São Carlos. Primeiramente é apresentado o processo de seleção do método de modelagem, diante dos principais métodos existentes. A seguir é apresentado o processo de desenvolvimento do modelo de referência, bem como as principais atividades e informações que o compõem, e finalmente é feita uma discussão sobre as possibilidades de uso do modelo, apresentando e mostrando uma aplicação para ensino.

\section{Modelagem de Processo de Negócio e Modelo de Referência}

Como dito anteriormente, processo de negócio representa o fluxo contínuo das atividades que acontecem nas empresas. Pelos processos de negócios pode-se materializar as políticas gerenciais, os fluxos de documentos e informações, os procedimentos operacionais e os processos de manufatura. E a maneira de se trabalhar orientado por processos de negócios é por meio da construção de um modelo dos processos de negócios da empresa.

Além do fato do modelo dos processos de negócios servir de base para implantação de ações de melhorias nas empresas, VERNADAT 
(1996) ressalta que a modelagem dos processos de negócios tem como finalidade:

- melhorar a representação e compreensão de como a empresa trabalha;

- racionalizar e assegurar o fluxo de informações;

- armazenar o conhecimento adquirido e o know how da empresa, para uso posterior;

- prover uma base para análises econômicas e organizacionais;

- simular o comportamento de partes da empresa;

- prover uma base para tomada de decisões operacionais e organizacionais e

- controlar, coordenar ou monitorar algumas partes da empresa (i.e., alguns processos).

Diante disso, o modelo dos processos de negócios torna-se relevante, uma vez que, a partir dele é possível construir uma visão única de aspectos comportamentais e organizacionais, podendo essa visão ser compartilhada por toda a empresa.

Para tanto o modelo deve conter diferentes representações, ser expressado por um formalismo, permitindo que, com certo grau de abstração as atividades, informações, recursos e a estrutura organizacional da empresa possam ser representadas por de uma linguagem (VERNADAT, 1996). As linguagens para modelagem podem ser definidas em termos de símbolos gráficos, textos ou até mesmo linguagem matemática dependendo do grau de formalismo desejado.

Atualmente existem várias arquiteturas de referência para o desenvolvimento do modelo dos processos de negócios, sendo que as que mais se destacam são as arquiteturas CIMOSA (Computer Integrated Manufacturing Open System Architecture) e ARIS (Architecture of Integrated Information System). O desenvolvimento desse trabalho utilizou a arquitetura ARIS.

A adoção da arquitetura ARIS como arquitetura de referência para o desenvolvimento do modelo apresentado, teve como principal aspecto o fato dessa arquitetura possuir uma ferramenta computacional capaz de contemplar todos os aspectos necessários para modelagem e ainda permitir a integração do modelo desenvol- vido com outros sistemas computacionais de apoio às atividades, tais como o sistema SAP $\mathrm{R} / 3$, processadores de textos e planilhas eletrônicas. Ou seja, pelo modelo, é possível acessar automaticamente informações que estão em um relatório, ou ainda processar transações do sistema SAP R/3. Essa característica é relevante pois torna o modelo mais didático e integrado com a forma de trabalho da empresa.

\subsection{ARIS - Architecture of Integrated Information Systems}

A arquitetura ARIS, cuja ferramenta possui o mesmo nome, utilizada para a descrição de processos de negócios, está dividida em quatro visões distintas: Organização, Função, Dados e Controle (SCHEER, 1991). Isto implica que cada processo é observado segundo uma perspectiva diferente para facilitar o entendimento. Para cada visão existem diferentes métodos que podem ser utilizados para a descrição da situação real.

A visão de dados representa todos os elementos que modificam ou geram dados, que são as condições e os eventos. Os eventos são representados por informações. Condições são referências, como por exemplo o status de uma ordem de fabricação, que também é um dado. Esta visão então é caracterizada por representar a movimentação (fluxo) de dados.

A visão de funções engloba as atividades e processos, apresenta uma descrição da função, a enumeração das suas subfunções (subprocessos) e o relacionamento entre as funções.

A visão organizacional representa as unidades organizacionais e usuários que compõem o processo. Devido à sua proximidade, estes dois elementos são representados juntos. Os usuários são associados a unidades organizacionais.

A integração entre as três diferentes visões ocorre pela visão de Controle. O método mais importante na visão de Controle é o EPC (Extended Event-Controlled Process Chain Method), que consiste num diagrama com dois elementos centrais: função e evento. $\mathrm{O}$ evento 


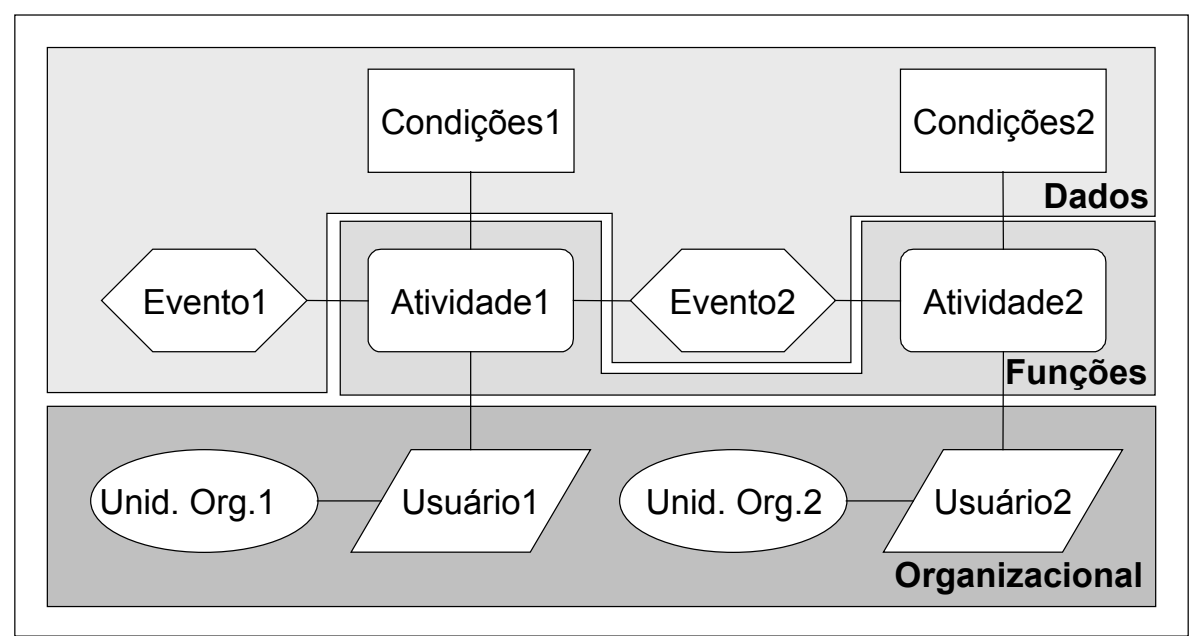

Figura 1 - Visões ARIS: dados, funções e organizacional.

contém informações que iniciam as funções, que por sua vez produzem eventos, e assim formando a seqüência de atividades que estão em um processo. Neste método, um processo é uma ocorrência, que é iniciada por um evento e finalizada por outro evento (Figura 1).

Como resultado obtém-se a descrição de todas as atividades do modelo de referência, bem como sua ordenação e relacionamento com cada área da empresa. Este método fornece vantagens na solução de problemas administrativos e organizacionais (SCHEER, 1998).

\subsection{Modelo de Referência}

Os modelos de referência, os quais podem ser desenvolvidos em situações reais ou em estudos teóricos, documentam os vários aspectos de um processo de negócio. Pode-se distinguir entre modelos procedimentais ou de implementação de softwares padrão, e modelos de negócios tais como modelos para gestão da produção e desenvolvimento de produtos (SCHEER, 1998). Além disso, os modelos de referência podem ser especializados para mercado, segmentos ou tipologias específicas, como por exemplo: setor automotivo, setor de alimentos, indústria aeroespacial e outros.

O objetivo do modelo de referência é prover a empresa com uma solução inicial para seus processos de negócios, para que, através dessa, seja especificado e detalhado o modelo particular da empresa.

Atualmente os principais fornecedores de modelos de referência são as empresas de consultoria e empresas de softwares corporativos. Essas empresas possuem modelos especializados para vários segmentos de mercado. Outro fornecedor de modelos de referência são os institutos de pesquisas e órgãos de pesquisa, porém os modelos tendem a ser mais genéricos e não tão detalhados.

Resumidamente, segundo VERNADAT (1996), as vantagens em se adotar modelos de referência consistem em:

- redução de tempo e custo no desenvolvimento do modelo particular;

- comparação das atividades da empresa com as atividades propostas no modelo (i.e., melhores práticas);

- melhor suporte na implantação de sistemas de gestão empresarial integrados.

\section{Modelo de Referência para Gestão da Produção - ATO}

desenvolvimento do modelo de referência em questão foi motivado pelas pesquisas desenvolvidas pelo Grupo de Logística Integrada - LI do Núcleo de Manufatura Avançada - 
NUMA. As atividades de pesquisa deste grupo tratam do planejamento, implementação, controle e otimização do fluxo físico e de informações da empresa e das cadeias de suprimentos nas quais atua, a partir dos objetivos estratégicos definidos. Para tal, engloba as áreas de planejamento e controle da produção, supply chain management e logística. Além disso existe o estudo sobre os sistemas ERP - Enterprise Resource Planning - como ferramenta para o auxílio à gestão da produção. O resultado dessas atividades é empregado em cursos para a comunidade acadêmica e empresarial bem como em projetos de extensão com empresas.

O processo de negócio de gestão da produção não ocorre de forma única nas empresas. Este deve ser modelado de acordo com as restrições dos diferentes tipos de sistemas produtivos. Segundo PIRES (1995), uma das maneiras de se diferenciar os sistemas produtivos é pelo grau com que o cliente final participa na definição do produto, sendo que as quatro tipologias de produção básicas são (Figura 2):

- Produção para Estoque (MTS - Make to Stock) - Caracteriza os sistemas que produzem produtos padronizados, baseados principalmente em previsões de demandas. Nesse caso, nenhum produto é customizado, porque o pedido é feito com base no estoque de produtos acabados. Isso significa que a interação direta dos clientes com o projeto dos produtos é muito pequena ou inexistente. Os sistemas MTS têm como principal vantagem a rapidez na entrega dos produtos, mas os custos com estoques tendem a ser grandes e os clientes não têm como expressar diretamente suas necessidades a respeito dos produtos. Nesses sistemas, os ciclos de vida dos produtos tendem a ser relativamente longos e previsíveis.

- Montagem sob Encomenda (ATO - Assembly to Order) - Caracteriza os sistemas em que os subconjuntos, grandes componentes e materiais diversos são armazenados até o recebimento dos pedidos dos clientes contendo as especificações dos produtos finais. A interação dos clientes com o projeto dos produtos é limitada. Nos sistemas ATO as entregas dos produtos tendem a ser de médio prazo e as incertezas da demanda (quanto ao mix e volume dos produtos) são gerenciadas pelo excesso no dimensionamento do estoque de subconjuntos e capacidade das áreas de montagem.

- Produção sob Encomenda (MTO - Make to Order) - O projeto básico pode ser desenvolvido a partir dos contatos iniciais com o cliente, mas a etapa de produção só se inicia após o recebimento formal do pedido. A interação com o cliente costuma a ser extensiva e o produto está sujeito a algumas modificações, mesmo durante a fase de produção. Num sistema MTO, os produtos geralmente não são um de cada tipo, porque usualmente os produtos são projetados a partir de especificações básicas. Os tempos de entrega tendem a ser de médio a longo prazo e as listas de materiais são usualmente únicas para cada produto.

- Engenharia sob Encomenda (ETO Engineering to Order) - É como se fosse uma extensão do MTO, com o projeto do produto sendo feito quase que totalmente baseado nas especificações do cliente. Os produtos são altamente customizados e o nível de interação com o cliente é muito grande.

Essas quatro tipologias básicas de produção definem ou direcionam grande parte das atividades que compõem o processo de Gestão da Produção. A escolha da tipologia ATO para o desenvolvimento do modelo de referência baseou-se no fato de que esta tipologia guarda características tanto da tipologia MTS como da tipologia MTO. Além disso, existe uma tendência das empresas que produzem exclusivamente sob encomenda, e das que produzem para estoque, de migração para a tipologia ATO. Isso porque as empresas que produzem para estoque tendem a ganhar com a possibilidade de fornecer um produto menos padronizado, que melhor atenda as necessidades dos clientes, e as empresas que trabalham exclusivamente sob 


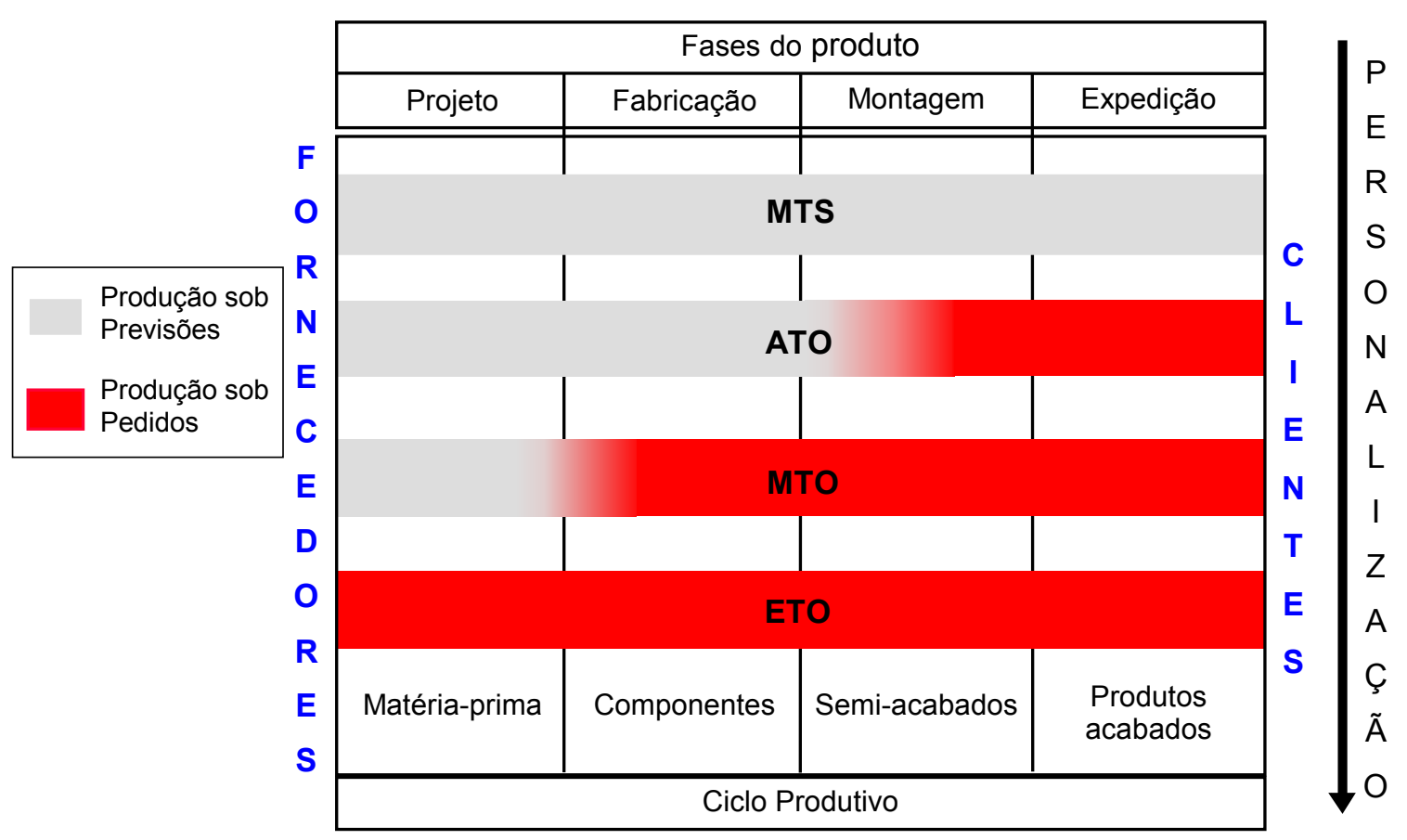

Figura 2 - Tipologias de produção.

encomenda têm a possibilidade de diminuir o prazo de fornecimento para seus clientes e tendem a ganhar também com a padronização de componentes de seus produtos finais.

\subsection{Processo de Desenvolvimento do Modelo de Referência}

O desenvolvimento do trabalho apresentado foi obtido por meio de pesquisas acadêmicas sobre gestão da produção, de trabalhos práticos desenvolvidos em empresas e, por último por entrevistas com empresas.

A etapa inicial do trabalho consistiu no levantamento das particularidades que tipologia de produção adotada impõe ao processo de gestão da produção. Uma vez concluída esta etapa, foram definidos quais os principais subprocessos (que também podem ser compreendidos como processos de negócios) que compõem o processo de gestão da produção. Esses subprocessos, que formam o nível mais abstrato do modelo, são: Gerenciar Demanda, Elaborar Planejamento Agregado da Produção, Elaborar Programa Mestre de Produção, Gerenciar Materiais,
Programar Produção e Controlar Produção. A Figura 3 mostra o diagrama de hierarquia de funções do ARIS, com o primeiro e o segundo níveis de detalhamento para o processo de negócio Gerenciar Demanda.

Após a definição dos subprocessos, passou-se então para o detalhamento de cada um deles, levantando principalmente as atividade $\mathrm{e}$ informações necessárias no modelo. A última atividade foi a junção das atividades e informações para o desenvolvimento do modelo EPC.

\subsection{Atividades e Informações do Modelo de Referência}

Nesse artigo mostraremos as principais funções e resultados que são obtidos dos seis subprocessos que compõem o processo de Gestão da Produção.

\subsubsection{Gerenciar Demanda}

As empresas precisam de mecanismos capazes de gerir sua demanda e necessitam gerir sua demanda. Isto é, precisam que as necessidades 


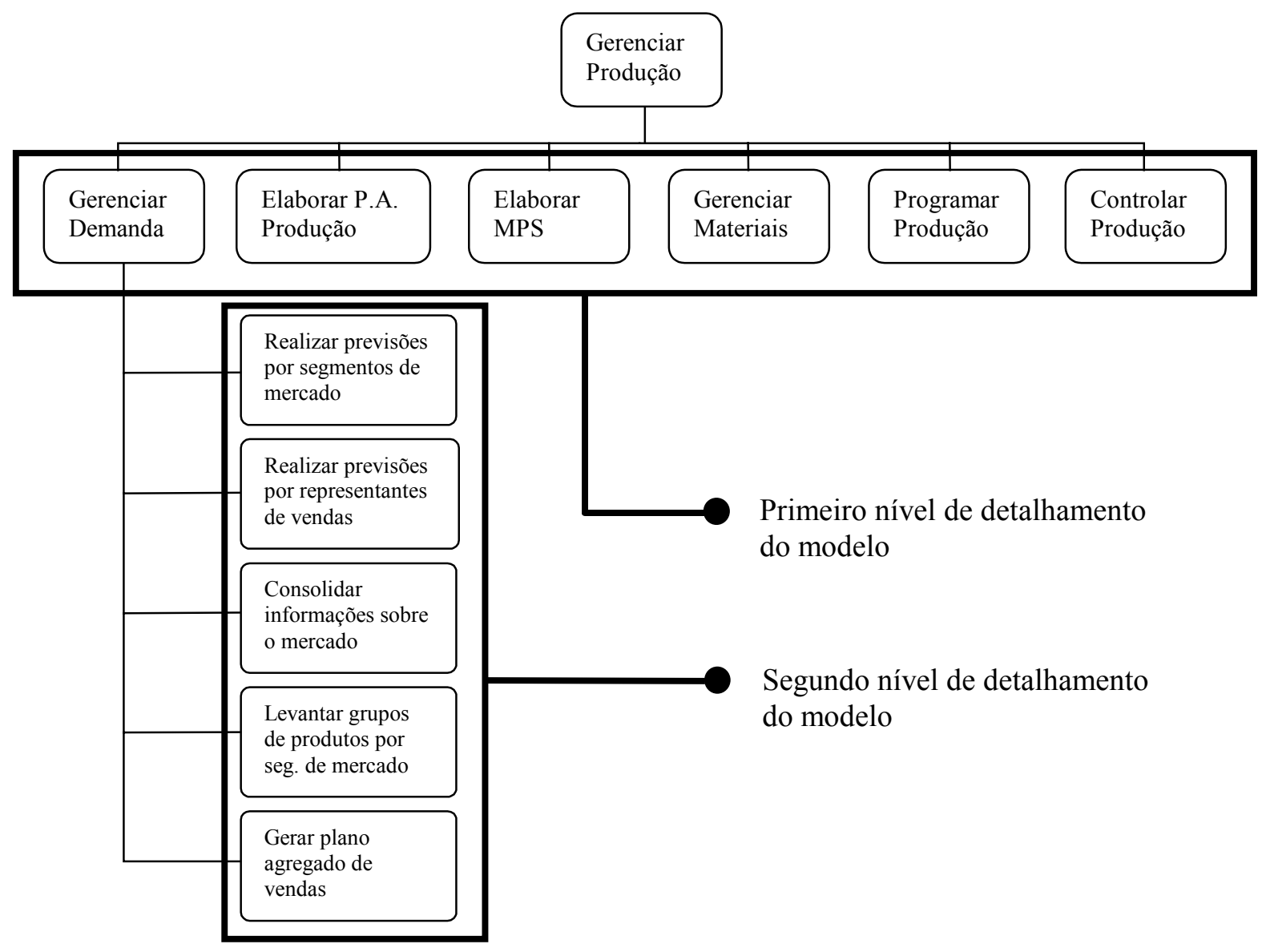

Figura 3 - Hierarquia de Funções.

dos clientes sejam determinadas e satisfeitas (VOLLMANN, 1993). O processo Gerenciar Demanda, busca prover a empresa de um conjunto de atividades capazes de gerir sua demanda, ou seja, identificar quais as necessidades dos clientes em termos de produtos e serviços, dimensionar a capacidade de absorção de cada segmento de mercado e segmento de cliente, e determinar qual o nível de atendimento para cada um desses segmentos.

Desse modo, o processo Gerenciar Demanda deve estar diretamente vinculado com o planejamento estratégico da empresa (VOLLMANN, 1997). Outra característica desse processo é que apesar de fazer parte do processo de Gestão da Produção, envolve informações e decisões financeiras, de vendas, da produção, da engenharia ou desenvolvimento de produtos, e de marketing. Isso faz com que o processo
Gerenciar Demanda seja realizado pelos gerentes e coordenadores dessas áreas da empresa, dando assim um caráter multidisciplinar a esse conjunto de atividades.

O principal resultado do processo Gerenciar Demanda é o plano agregado de vendas. Esse plano contém uma meta de vendas da empresa para um determinado período. Esse é um período longo, geralmente de um ano. O plano agregado de vendas, como o próprio nome diz, fornece as quantidades para vendas em termos agregados, ou seja, em função de valores financeiros, ou então para grupos ou famílias de produtos, ou ainda por segmentos de mercado. Essas informações são extremamente importantes para a produção, e devem respeitar as metas estabelecidas no planejamento estratégico da empresa. Desse modo o plano agregado consegue atender uma característica da tipologia 
ATO, que é a impossibilidade de realizar um plano de vendas para cada produto específico. E, em alguns casos ainda, as empresas que adotam essa tipologia possuem um grande número de produtos finais.

Para gerar o plano agregado de vendas são realizadas atividades como: previsões estatísticas de vendas por segmentos de mercado, análise de fatores econômicos para cada um dos segmentos de mercado, pesquisa por representantes de vendas, análise financeira de cada grupo de produtos, levantamento de novos produtos a serem lançados, levantamento das promoções de vendas planejadas, entre outras. Métodos e técnicas devem ser associados a cada uma dessas atividades. Cada análise e atividade realizadas devem conter ainda um fator de relevância, cujo objetivo é indicar quão bem a empresa executa essas atividades. Esse fator de relevância é tomado com base no histórico de realização da atividade específica. Quanto maior o fator de relevância, maior o peso da atividade para a consolidação do plano agregado de vendas. Esse plano deve ser revisto periodicamente ao longo do ano, para que seja realizado um controle daquilo que foi planejado e para que novos fatores que possam alterar o comportamento das vendas sejam incluídos.

As principais informações utilizadas nessa parte do processo são: histórico de vendas, histórico com comportamento dos segmentos de mercado, relatórios de análise financeira por grupo de produtos, relatórios de pesquisa de mercado, histórico de faturamento, relatório de investimento nos grupos de produtos.

\subsubsection{Elaborar Plano Agregado de Produção}

Uma vez gerado o plano agregado de vendas, deve-se convertê-lo, com base em vários fatores, num plano agregado de produção, que servirá de meta para a manufatura. Essa é umas das principais funções do plano agregado de produção.

As decisões financeiras, de vendas, de engenharia são integradas à decisão de manufatura pelo plano de produção. O plano de produção é, portanto um fator chave para coordenar as decisões na empresa (VOLLMANN, 1993). Além disso, procura determinar os recursos necessários para executar os planos estratégicos da empresa (SKINNER, 1978). Ele reflete ainda o posicionamento da alta gerência no que se refere à direção a ser tomada para os negócios, bem como o impacto das influências macroeconômicas. Portanto ele é diretamente relacionado com o plano agregado de vendas.

Porém, o plano agregado de produção não é uma previsão da demanda. É uma produção planejada, feita numa base agregada, pela qual a gerência de manufatura deve ser responsável por cumprir. Também o plano agregado de produção não é necessariamente igual ao plano agregado de demanda.

Durante a conversão do plano agregado de vendas para o plano agregado de produção, a empresa tem a possibilidade de inserir parâmetros associados à estratégia de manufatura e à capacidade de produção, podendo se antecipar à demanda caso seja necessário, ou adequado, devido à tipologia de produção adotada pela empresa. Para tanto, a capacidade deve ser analisada em termos agregados, levantando-se o número de horas disponíveis em linhas de produção, unidades fabris, centros de trabalhos e mão-de-obra. Essa análise da capacidade tende a restringir o plano agregado de produção, ou direcionar recursos para o aumento da capacidade produtiva.

Uma vez gerado o plano agregado de produção, esse deve ser desagregado para os níveis inferiores de planejamento. Esses níveis inferiores de planejamento podem ser subfamílias de produtos, produtos finais, unidades fabris ou linhas de produção. A finalidade dessa atividade é distribuir as metas de produção para cada unidade representativa de produção.

\subsubsection{Elaborar Programa Mestre de Produção}

O processo Elaborar Programa Mestre da Produção tem como objetivo desenvolver o programa mestre da produção. Esse programa é 
um planejamento da produção de curto/médio prazo, que considera os pedidos existentes e é realizado em função dos produtos finais e componentes críticos, e não mais em termos agregados como no plano agregado de produção. Porém, o programa mestre de produção deve respeitar e considerar as decisões tomadas no plano agregado de produção.

O programa mestre de produção executa também a função de conciliar a demanda existente e a capacidade disponível para aquele período, procurando atender os pedidos dentro dos prazos estabelecidos e prever os momentos em que irá ocorrer o atraso de um pedido, tornando possível assim uma negociação com o cliente.

As principais informações utilizadas nessas atividades são: pedidos em carteira, pedidos atrasados, capacidade disponível, produtos e lista de materiais, pedidos programados, entre outras. O programa mestre de produção permite a simulação de situações de planejamento da linha de montagem onde é possível medir a quantidade de estoque gerado, o número de pedidos atrasados, o custo médio do produto fabricado. Com base nessas simulações é escolhida a melhor programação dos pedidos na fábrica.

Uma atividade importante desse subprocesso é a gestão do pedido. Esta visa garantir o atendimento de um pedido desde o processo de vendas. Pela verificação automática da disponibilidade de materiais e capacidade durante a entrada do pedido, é possível saber se empresa é capaz ou não de atender o pedido dentro do prazo estipulado pelo cliente; caso seja possível o pedido é aceito e automaticamente e gera-se uma ordem de montagem do produto final, caso contrário deve-se sugerir a partir de que data o pedido poderá ser aceito.

\subsubsection{Gerenciar Materiais}

$\mathrm{O}$ processo Gerenciar Materiais executa principalmente dois conjuntos de atividades. $\mathrm{O}$ primeiro, refere-se ao estabelecimento de uma política de estoque para cada item envolvido no processo produtivo e o segundo, refere-se ao cálculo da necessidade líquida de cada item dentro de um determinado período.

$\mathrm{O}$ estabelecimento da política de estoque do item visa estabelecer como será o planejamento e controle de estoque do mesmo, como por exemplo: ponto de reposição, MRP (Manufacturing Requirements Planning), entre outros. Vários fatores influenciam no estabelecimento desta política de estoque, tais como: característica do fornecimento, custo de fabricação ou de compra, consumo médio do item, importância do item na composição do produto final.

$\mathrm{O}$ cálculo das necessidades líquidas é feito com base na política de armazenamento dos itens, e a partir desse cálculo é que são geradas as ordens de produção de componentes para o chão-de-fábrica. As informações para essa fase do processo são: o consumo dos itens, lista de materiais do item, custo de fabricação, custo de armazenagem e o tempo de suprimento.

\subsubsection{Programar Produção}

As atividades de programação e controle da produção estão relacionadas com o gerenciamento das atividades, passo a passo, envolvidas no processo produtivo. Production Activity Control (PAC), como é chamado em inglês, visa direcionar a programação detalhada e controlar as tarefas individuais nos centros de trabalho (VOLLMANN, 1993).

O processo de programação e controle da produção executa a gestão do nível mais detalhado do processo de gestão da produção. Está relacionado com a execução dos planos realizados nos processos anteriores. De modo geral, o processo de programação está relacionado com: o registro gerado pelo cálculo das necessidades de materiais, as ordens liberadas, o plano de capacidade, o roteiro de produção, o status da ordem, a performance do chão-de-fábrica.

$\mathrm{O}$ planejamento detalhado de materiais gera as necessidades a serem fabricadas, e o plano de capacidade mostra a capacidade disponível para a programação. Essas necessidades são então convertidas em ordens de produção e requisições 
de compras, e liberadas para o chão-de-fábrica e compras respectivamente. Durante a liberação das ordens de produção, é executada novamente uma verificação da disponibilidade de capacidade e materiais e ainda é feita a reserva da capacidade e dos materiais para aquela ordem de produção. Essas ordens são gerenciadas no chãode-fábrica por um sistema de programação da produção.

Para tanto, os sistemas de programação da produção utilizam algoritmos matemáticos de seqüenciamento de operações em máquinas. Isso faz com que o uso de sistemas computacionais de suporte seja indispensável. Os algoritmos de programação visam diminuir o tempo de processo da ordem, o tempo de fila, o tempo de transporte, entre outros, otimizando assim a capacidade de produção disponível.

\subsubsection{Controlar Produção}

As atividades do subprocesso de controle da produção visam monitorar o fluxo de trabalho e o consumo de materiais de tempo no chão-defábrica, bem como verificar se os componentes estão de acordo com os padrões de qualidade estabelecidos. As atividades dessa fase do processo são responsáveis por promover o feedback de informações para os processos descritos anteriormente.

As atividades de controle da produção iniciam-se ao final de uma operação e referem-se ao apontamento de informações no sistema. São apontados o tempo gasto com o setup da máquina, o tempo de processo da operação, o número de peças consumidas durante setup, a quantidade de matéria-prima consumida na operação, o número de peças produzidas e $\mathrm{o}$ número de peças produzidas com defeito. Com essas informações é possível calcular mais precisamente o custo de produção. Além disso, essas informações atualizam os estoques, os históricos de consumo de matérias-primas, e a capacidade disponível de produção, podendo também informar se uma ordem está ou não dentro do prazo estabelecido para sua conclusão.
A atividade de controle da qualidade também é realizada após a operação, sendo responsável por verificar se a peça atende as especificações. Isso é feito por dispositivos e instrumentos de medição. Uma vez identificado um defeito ou problema, esse deve ser relatado em laudospadrão. Esses laudos são emitidos por pessoas capacitadas da área de qualidade, e também devem ser inseridos no sistema de controle.

\section{Aplicações do Modelo de Referência Desenvolvido}

U $\mathrm{m}$ aspecto importante na implantação de um modelo de referência em uma empresa é a definição do objetivo ao qual destina-se esse modelo. Como dito anteriormente o modelo de referência fornece à empresa uma solução inicial de processos de negócios, porém cabe a empresa determinar quais são os processos que mais se assemelham com as suas operações, e ainda com que grau de detalhamento a empresa irá construir seu próprio modelo. Isso está diretamente relacionado com os objetivos da empresa para com os modelos de referência. A empresa, diante de seus objetivos, pode desenvolver seu modelo particular priorizando cada uma das visões do modelo.

O modelo apresentado dá maior ênfase às informações e atividades que compõem o processo de Gestão da Produção, isso porque tem como objetivos principais o suporte a implantação de sistemas ERP, suporte a gestão de conhecimento necessário para o processo de gestão da produção e o auxílio ao ensino de gestão da produção.

\subsection{Suporte a Implantação de Sistemas ERP}

Atualmente, presenciamos um crescente aumento nas implantações de sistema de gestão empresarial integrado, também chamado de Enterprise Resoucer Planning - ERP. Uma característica importante do processo de implantação desses sistemas é volume de informações referentes a empresa que é movimentado. 
O foco desses sistemas é atender os objetivos estratégicos da empresa e otimizar a execução das atividades do negócio (KIRCHMER, 1998). Para tanto se faz necessário o levantamento dos principais processos de negócios existentes na empresa, com ênfase nas informações processadas, nas atividades e na estrutura organizacional da empresa.

O uso de processos de negócio na implantação de sistemas ERP é possibilitado pelas características de integração, descentralização, dinamicidade, e orientação ao usuário existentes nos sistemas ERP. Características essas, típicas de uma estrutura orientada a processo de negócios.

Desse modo, é necessário o levantamento dos processos apropriados para se atingir os objetivos estratégicos da implantação. Esses processos devem receber o suporte dos sistemas ERP, através dos seus diversos módulos e, por fim, o usuário deve ser capacitado para execução desses processos pelo uso dos sistemas.

O modelo apresentado por esse trabalho está sendo utilizado para dar suporte a implantação do sistema SAP R/3 no NUMA, no âmbito das pesquisas desenvolvidas pelo Grupo de Logística Integrada. Para tanto, foi criada uma empresa fictícia, cuja tipologia de produção é a mesma do modelo de referência em questão, e ainda adota integralmente o modelo de referência como seu modelo particular. Ao final dá implantação, espera-se ter o modelo validado para dar suporte à implantação de sistemas ERP.

\subsection{Gestão do Conhecimento Necessário para o Processo de Gestão da Produção}

O conhecimento corporativo é cada vez mais relacionado como um importante componente para a gestão dos negócios da empresa. Este inclui os conhecimentos referentes aos produtos, tecnologias, procedimentos organizacionais, bem como o conhecimento de cada indivíduo que compõe a organização.

A documentação, armazenagem e utilização desse conhecimento são as atividades-chave do processo de gestão do conhecimento (SCHEER, 1998). Pelas informações existentes no modelo de referência é possível a documentação e armazenagem do conhecimento utilizado pela empresa. As atividades provêm um mapa de como as informações são utilizadas e transformadas, e ainda, para execução de determinadas atividades é necessário que os usuários tenham conhecimentos específicos requeridos pela atividade. Pela ligação da estrutura de atividade do modelo de referência com a estrutura organizacional é possível identificar as pessoas habilitadas com os conhecimentos necessários para execução de uma determinada atividade, bem como planejar o desenvolvimento de determinado conhecimento na organização.

Isso faz com que o modelo de referência seja uma importante ferramenta para a gestão do conhecimento, uma vez que esta é capaz de armazenar e documentar os conhecimentos existentes na organização, e ainda servir de base para planejar o desenvolvimento de novos conhecimentos, sempre sendo norteado pelos objetivos estratégicos da empresa.

\subsection{Ensino de Gestão da Produção}

Outra aplicação encontrada para o modelo de referência é o ensino de gestão da produção. Essa aplicação foi testada e validada dentro da disciplina de Planejamento e Controle da Produção II do curso de graduação em Engenharia de Produção Mecânica da Escola de Engenharia de São Carlos.

A proposta do uso do modelo para ensino consiste em duas etapas. Na etapa inicial do curso, os conhecimentos referentes à gestão da produção são transmitidos da maneira tradicional, expositiva. Numa segunda fase é exigido que os alunos, organizados em grupos de trabalho, construam seus próprios modelos, com base numa situação dada. Esses modelos são então comparados com o modelo de referência para possíveis correções e esclarecimento de dúvidas.

A parte mais importante é quando os alunos têm que construir seus próprios modelos. A 


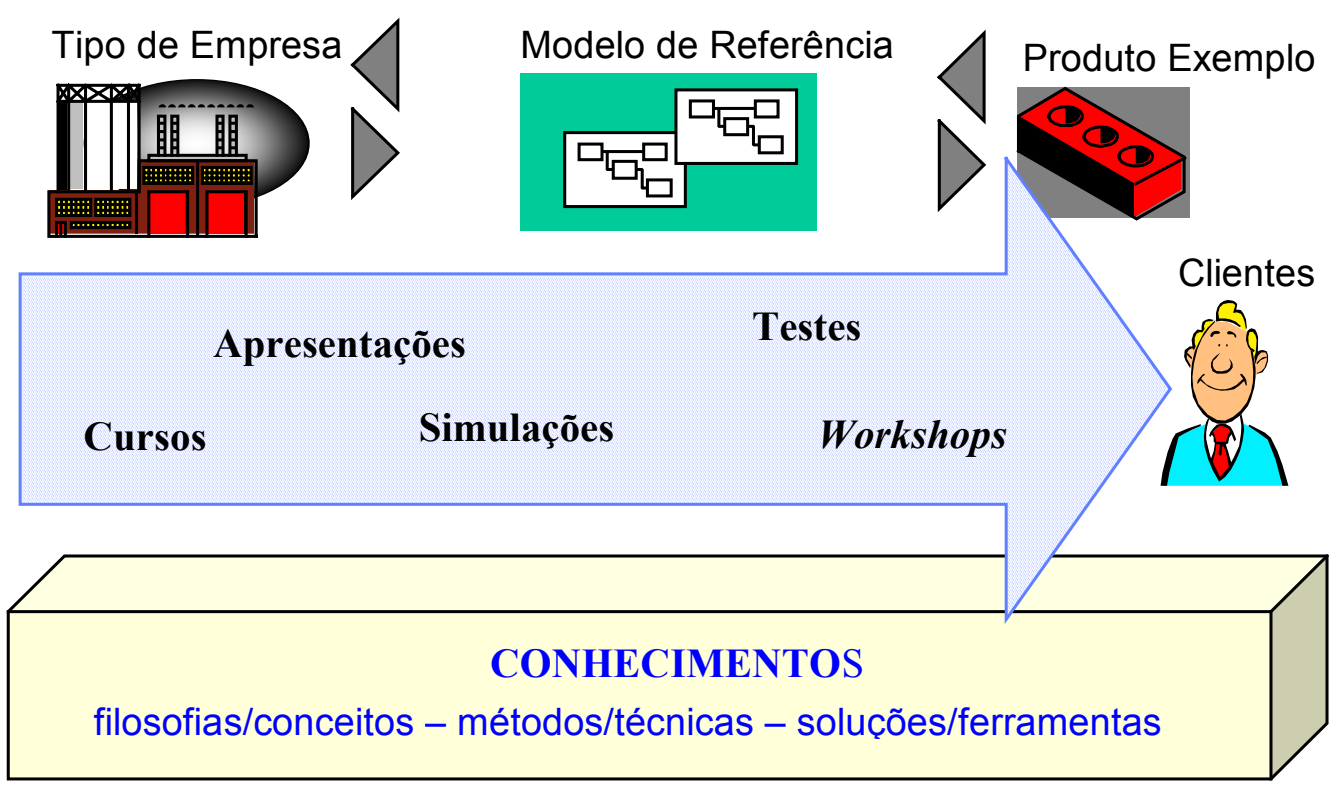

Figura 4 - Estrutura e Aplicações do Cenário.

partir dessa atividade geram-se discussões importantes para o aprendizado, e além disso, o modelo permite uma melhor compreensão de cada uma das fases da gestão da produção, e da relação existente entre elas, bem como a importância de conceitos, métodos e técnicas para a realização de cada uma dessas fases.

Além da aplicação nas disciplinas, foi desenvolvido um ambiente que possibilita o aprendizado de forma prática, onde o modelo de referência é um dos componentes desse ambiente (LENZA, 1999). A esse ambiente é dado o nome de cenário. O cenário tem como objetivo servir de base para o aprendizado dos conhecimentos a partir de uma maneira prática, onde o aluno pode vivenciar o conhecimento aplicado que se espera transmitir. Para tanto, o cenário é constituído dos seguintes elementos: produto exemplo, tipo de empresa, base de conhecimentos e um modelo de referência. Estes elementos estão detalhados na Figura 4.

Modelo de Referência: para que a empresa possa ter uma visão por processos de negócios é necessário que os mesmos sejam identificados, mapeados e representados, tornando possível uma compreensão das atividades, informações, recursos que existem na organização. O modelo de referência é o instrumento utilizado para representar os processos de negócios. Para que as atividades do modelo de referência possam ser realizadas é necessário o domínio de conhecimentos específicos.

Base de Conhecimento: a base de conhecimento reúne um conjunto de conhecimentos relativos a um determinado assunto. Esses conhecimentos podem ser sistematizados da seguinte forma:

- Conceitos/Filosofias: são os conhecimentos mais amplos que servem para criar um embasamento teórico e fornecer diretrizes para a implantação dos métodos, técnicas, sistemas, ferramentas e soluções.

- Técnicas/Métodos: são conhecimentos não tão abrangentes como os conceitos e filosofias e normalmente são estruturados em passos, ou relacionados com algo específico, para atingir um determinado objetivo. Muitas vezes eles relacionam-se com os conceitos e filosofias, podendo até ser classificados como tal.

- Ferramentas/Sistemas: são conhecimentos relacionados com produtos comerciais, que podem ser utilizados nos processos. Normalmente, uma ferramenta está associada a um conceito e/ou método. 
Tipo de Empresa: procura definir o ambiente para o qual foi desenvolvido o modelo de referência. Fatores como porte, área de atuação, segmento de mercado, produtos, fornecedores ajudam a caracterizar um tipo de empresa. $\mathrm{Ou}$ seja, para cada configuração que se cria, como por exemplo: uma empresa de grande porte, que atua tanto no mercado interno como regionalmente, dentro do segmento automotivo; tem-se um modelo de referência que é mais adequado.

Tipo de Produto: tem como finalidade caracterizar o produto desenvolvido e comercializado pela empresa. De acordo com o tipo de produtos, tem as definições de como é o sistema produtivo dessa empresa, como é o relacionamento com seus fornecedores e clientes, e quais são as tecnologias envolvidas no seu processo de fabricação.
A relação existente entre esses quatro elementos é que o tipo de empresa e o tipo de produto dão as restrições necessárias para o desenvolvimento do modelo de referência. Associados ao modelo desenvolvido estão os conhecimentos necessários para a realização das atividades.

Além desses quatro elementos é preciso prover o cenário de uma infra-estrutura capaz de suportar a aplicação disso tudo. Essa infraestrutura deve ser composta por hardwares, softwares, recursos áudio visuais capazes de suportar realização das atividades do modelo de referência.

Desse modo, tem-se um ambiente onde é possível transmitir conhecimento com maior nível de absorção, ou seja, pela vivência dos conhecimentos necessários para realização das atividades do modelo de referência para um determinado tipo de empresa e produto.

\section{Referências Bibliográficas}

LENZA, R.P.; BREMER, C.F. \& GIL, L.F.A.: "Desenvolvimento de um cenário para ensino de Gestão da Produção." Anais do XIX Encontro Nacional de Engenharia de Produção, Brazil Fourth Congress of Industrial Engineering, ENEGEP, Universidade Federal do Rio de Janeiro, Nov. 4-8, 1999.

KIRCHMER, M.: Business Process Oriented Implementation of Standard Software, SpringerVerlag, Berlin, 1998.

PIRES, S.R.I.: Gestão Estratégica da Manufatura, Editora UNIMEP, 1995.

ROZENFELD, H.: "Reflexões sobre a Manufatura Integrada por Computador (CIM)", Manufatura Classe Mundial: Mitos e Realidade, São Paulo, 1996.

SCHEER, A.W.: Business Process Frameworks, Springer-Verlag, Berlin, 1998.
SCHEER, A.W.: Business Process Engineering Reference Models for Industrial Enterprises, Springer-Verlag, Berlin, 1994.

SCHEER, A.W.: Principles of Efficient Information Management, Springer-Verlag, Berlin, 1991.

SKINNER, C.W.: Manufacturing in the Corporate Strategy, Wiley, New York, 1978.

VERNADAT, F.B.: Enterprise Modeling and Integration: principles and applications, Chapman \& Hall, London, 1996.

VOLLMANN, T.E.: Integrated Production and Inventory Management, Business One Irwin, Homewood, 1993.

VOLLMANN, T.E.; BERRY, W.L. \& WHYBARK, D.C.: Manufacturing Planning and Control System, McGraw-Hill, New York, 1997. 


\title{
A REFERENCE MODEL FOR PRODUCTION MANAGEMENT IN ASSEMBLY TO ORDER - ATO PRODUCTION SYSTEMS AND ITS MULTIPLE APPLICATIONS
}

\begin{abstract}
The search for competitiveness in the companies heads each time more for a business process oriented structure. Ahead of this, the reference models came up with a relevant importance, because they are responsible for documenting and mapping the business processes. This article presents a reference model for the business process of Production Management, applied for assembly to order enterprises. This reference model was developed by the Integrated Logistic Group from the Nucleus of Advanced Manufacturing from USP - São Carlos. Initially the selection process of the modeling method is presented, ahead of the main existing methods. After this, the development process of the reference model is presented, as well as the main activities and information that compose it and finally a discussion about the possibilities of the use of the model presented is made and an application for education is shown.
\end{abstract}

Key words: production management, reference model, business process. 